\title{
ESTADO Y MUNICIPIO
}

342.4: 352 (892. Paraguay)

$$
\text { por }
$$

\section{Luis-Enrique Chase Plate \\ Profesor de Derecho Administrativo de la Facultad de Derecho y Ciencias Sociales de la Universidad Nacional de Asunción}

SUMARIO: I. EL ESTADO.-II. FORMACION DEL ESTADO PARAGUAYO: 1. El REglaMENTO DE GOBIERNO DE 1813. 2. LA CONSTITUCIÓN DE 1844. 3. LA CONSTITUCIÓN DE 1870.-III. LA CONSTITUCION DE 1967.-IV. DIVISION DE PODERES.-V. PODER LEGISLATIVO. - VI. PODER EJECUTIVO. - VII. PODER JUDICIAL: 1. TRIbunal DE CuENTAS. 2. Recurso DE INCONSTitucionaLIDAD. 3. JERARQuía de los ÓRganos JUdiciales. 4. El Ministerio PÚBLICO.-VIII. EL MUNICIPIO PARAGUAYO.-IX. LA AUTONOMIA MUNICIPAL EN LA CONSTITUCION.-X. LEYES MUNICIPALES: 1. LA LEY NÚ MERO 915. 2. LEY NÚMERO 222 ORGÁNICA MUNICIPAL. - XI. PRESCRIPCIONES CONSTITUCIONALES. XII. ELEMENTOS ESENCIALES.

\section{EL ESTADO}

El Estado es la sociedad jurídicamente organizada. Desde tiempos remotos el hombre buscó afanosamente un instrumento adecuado y efectivo para poner orden y organizar la sociedad, creándose así, con el devenir de los siglos, lo que hoy se denomina Estado. 
A través de la historia se han ido elaborando diversas concepciones doctrinarias sobre el Estado.

SOLER (1) considera a la Nación como substractum del Estado.

El gran filósofo Jacques Maritain explica con claridad las dos grandes teorías que existen sobre el Estado. La teoría instrumentalista, a la que podemos señalar como la concepción de un Estado al servicio del hombre, en lo que en modo alguno el hombre es para el Estado, sino el Estado para el hombre. Pero entiéndase bien que esto no quiere decir que al Estado hay que sacarle la autoridad que debe tener, sino que esa autoridad máxima, ese poder o imperio debe estar al servicio del bien común.

MARITAIN (2) expresa cuando se refiere al Estado que «éste es sólo aquella parte del cuerpo político especialmente interesada en el mantenimiento de la Ley, el fomento del bienestar común y el orden público, así como la administración de los asuntos públicos. El Estado es una parte que se especializa en los intereses del todo. No es un hombre ni un conjunto de hombres; es un haz de instituciones combinadas que forman una máquina situada en la cima: este tipo de obra de arte ha sido construida por el hombre y utiliza cerebros y energías humanas, y no es sino hombre, pero constituye una encarnación suprema de la razón, una superestructura impersonal y pervivente, cuyo funcionamiento se podría calificar de racional en segundo grado, dada la actividad de la razón que contiene, pero que, limitada por la Ley y por un sistema de reglamentaciones universales, es más abstracta, más alejada de las contingencias de la experiencia y también más despiadada que nuestras vidas individuales». "El Estado no es la encarnación suprema de la Idea, como creía HEGEL; ni tampoco una especie de superhombre colectivo; el Estado no es sino un organismo facultado para utilizar el poder y la coerción, integrado por expertos o especialistas en ordenamiento y bienestar públicos: un instrumento al servicio del hombre. Poner el hombre al servicio de ese instrumento es perversión política. El ser humano como individuo es para el cuerpo político, y el cuerpo político es para el ser humano como persona. Pero en modo alguno el hombre es para el Estado, sino el Estado para el hombre».

Hemos creído oportuno ubicar al Estado en el contexto de este estudio, pues de la noción que se tenga de él resultan los principios

(1) Juan-José Soler: Introducción al estudio del Derecho, pág. 110.

(2) Jacques Maritain: El hombre y el Estado, Buenos Aires, 1952, pág. 25. 
cardinales que marcan toda acción humana. Un proyecto de Ley o una Ley pueden obedecer en una etapa histórica a una determinada filosofía jurídica. De la idea o el concepto que tengamos del Estado nacerá nuestra forma de dar las respuestas a los planteamientos de nuestro tiempo. Este no es un estudio de ciencia política o de Derecho político, ni pretende serlo. Pero entendemos que la filosofía del Derecho trasunta la necesidad humana de investigar el valor justicia, poniendo en constante evaluación el ser con el deber ser jurídico. No debe olvidarse en ninguna construcción jurídica la «verdad ideal» ni la «realidad empírica»; ambas contribuyen a la formación de la juridicidad desde su territorio insoslayable.

El hombre es el creador y protagonista de la historia. «El Estado da vida a la Nación»; es un instrumento formidable para guiar la comunidad nacional hacia senderos de concordia, paz y felicidad.

La sociedad no puede vivir sin autoridad ni poder. La vida social se basa sobre una organización, pues necesita de esa organización para subsistir y convivir.

Sin orden social y paz pública la sociedad sería un caos; imperaría la anarquía, en la que se impondría la fuerza del más fuerte sobre el más débil, y se estaría en el estadio de las hordas. El poder y la coerción del Estado obedecen a una necesidad de la vida humana. Sin ese poder se establecería la violencia y el desorden, regresando al estado de naturaleza.

Ludwig von Mises (3), partiendo de lo que es la naturaleza humana, considera al Estado como una institución necesaria e indispensable y, adecuadamente administrada, base de la sociedad, de la cooperación humana y de la civilización. Expresa von Mises que el Estado «es el instrumento más beneficioso y más útil que ha encontrado el hombre en sus esfuerzos para promover la felicidad y el bienestar de la humanidad. Pero es únicamente un instrumento, un medio, y no un fin. No es Dios. Es simplemente compulsión y coerción, fuerza policial».

Consideramos nosotros que el Estado es una institución ideal creada por el hombre y que debe estar a su servicio. No es el Estado el que crea al hombre, sino a la inversa, de manera a dotar a la sociedad y a la Nación del aparato jurídico-político que regule armoniosamente la convivencia y la tolerancia humana. La desvirtuación de la noción del Estado ha causado ya a la humanidad grandes guerras y conflictos de todo orden.

(3) Ludwig von Mises: Omnipotencia gubernamental, pág. 85. 
No se debería pretender la constitución de un Estado perfecto. No creemos que esto debe ser el propósito, sino a la inversa, como bien lo enseña ORTEGA y GASSET (4) cuando expresa que ve aque casi todo el mundo - autoritarios como radicales- moviliza su intelecto en esta falsa dirección: ¿cómo es posible crear un Estado lo más perfecto que quepa imaginar? (Para el autoritario y para el radical la perfección del Estado consiste en cualidades divergentes; pero el propósito es común: lograr un Estado perfecto)». El genial pensador señala que «para quien piensa que la perfección del Estado se halla fuera de él, en la perfección del cuerpo nacional, el pensamiento político tiene que volver del revés la cuestión: ¿cómo hay que organizar el Estado para que la Nación se perfeccione?» Sigue diciendo que, «en definitiva, quien vive es la Nación. El Estado mismo, que tan fecundamente puede actuar sobre ella, se nutre, a la larga, de sus jugos».

Expresa que "cabría decir que un Estado es perfecto cuando, concediéndose a sí mismo el mínimo de ventajas imprescindibles, contribuye a aumentar la vitalidad de los ciudadanos». «En la historia triunfa la vitalidad de las Naciones, no la perfección formal de los Estados».

En este mismo sentido coincide el maestro Franco Sobrin h o (5) cuando dice que «de modo absoluto el Estado está dentro de la Nación. Organízase para la Nación. Actúa soberanamente en nombre de la Nación». Expresa que «argumentar en otro sentido será lo mismo que jugar con abstracciones».

Manuel Garcfa Pelayo (6) explica en una condensación admirable lo que es el Estado y sus fines. Dice el destacado maestro «que el Estado es una organización que tiene por objeto asegurar la convivencia pacífica y la vida histórica de un grupo humano. Pacífica no quiere decir basada en el consentimiento general, sino simplemente en la eliminación de la violencia en las relaciones entre los individuos y grupos que forman la población de un Estado; vida histórica significa decidir su propio destino y según las posibilidades de una situación». Más adelante agrega: «Por consiguiente, y en todo caso, el Estado se manifiesta como una unidad de poder. Mas tal

(4) José Ortega y Gasset: «Mirabeau o el político», Obras Completas, tomo III, Revista de Occidente, Madrid, pág. 631.

(5) Manuel de Oliveira Franco Sobrinho: Curso de Direito Administrativo, Brasil, 1979, pág. 3 .

(6) Manuel Garcí Pelayo: Derecho Constitucional Comparado, Madrid, Revista de Occidente, pág. 19. 
poder necesita ser ejercido por alguien y, para ser eficaz, estar organizado según ciertas reglas. En consecuencia, es esencial a la vida del Estado establecer: a) quiénes están llamados a ejercer su poder; b) con arreglo a qué principios orgánicos; c) según qué métodos; d) con qué limitaciones. El contenido de estas reglas, en cuanto que se reputen obligatorias, forma el Derecho constitucional. Así pues, éste se nos aparece como parte integrante necesaria de la organización estatal».

El distinguido tratadista Jaime Vidal PERDomo (7), en su valioso libro Derecho Constitucional General, expresa las nociones generales sobre el Estado. Analiza en qué momento se forma el Estado y las condiciones de la existencia del mismo. Del mismo modo, Julio A. PRAT (8) explica con certera claridad y profundo análisis lo que es el Estado de Derecho y los cometidos y funciones del Estado.

\section{FORMACION DEL ESTADO PARAGUAYO}

El Paraguay nació como Estado independiente en 1811. Su primer Gobierno fue pluripersonal, configurado en una Junta, como en la mayoría de los países del Continente latinoamericano.

En los primeros años se alternó el triunvirato, el sistema bipersonal y el unipersonal, toda una gama de Gobiernos sin Constitución. Aunque el primer Gobierno independiente de la Provincia haya gobernado sin Carta política, el Paraguay ya constituía una Nación en una etapa avanzada de su evolución social con todos los elementos propios que requieren un Estado. A partir de 1811, el Paraguay tuvo cuatro instrumentos jurídicos fundamentales que organizaron el Estado y determinaron un orden jurídico.

\section{El Reglamento de Gobierno de $\mathbf{1 8 1 3}$}

Un Congreso constituido por más de mil diputados lo sanciona por aclamación. No constituye una Constitución propiamente dicha, sino un mero Reglamento de gobierno que instaura la institución del Consulado, designando a dos Cónsules de la República del Pa-

(7) Jaime Vidal Perdomo: Derecho Constitucional General, Bogotá (Colombia), 1978.

(8) Julio A. Prat: Derecho Administrativo, tomo 1. 
raguay, siendo ésta la primera República proclamada en Sudamérica. Se crea un sistema de gobierno - el Consulado- que provenía de Roma.

\section{La CONSTitución DE 1844}

El Congreso de 1844 sanciona una Constitución denominada «Ley que establece la Administración política de la República del Paraguay». Esta Constitución, a la que don Carlos Antonio LÓPEz llamó simplemente Ley, establecía una división de los Poderes ejecutivo, legislativo y judicial. La Constitución garantiza la independencia del Poder judicial. Tuvo vigencia hasta el año 1870.

\section{La CONSTitución DE $\mathbf{1 8 7 0}$}

La guerra había aniquilado al Paraguay. De 1.300.000 habitantes con que contaba el país, apenas 200.000 quedaron para restablecer las heridas de la Patria, en su mayoría mujeres, niños y ancianos. Esta Constitución, a la que un autor calificó como una de las más liberales de América, estuvo inspirada en la Constitución argentina de 1853 y algunos de sus artículos copiados textualmente. Dadas las circunstancias de aquella época, la Constitución del 70 no fue una expresión jurídica del pueblo paraguayo. En febrero de 1940 se deroga la Constitución de 1870 y se dicta la Constitución de 1940, que es luego sometida a un veredicto popular. Tiene vigencia hasta el año 1967.

\section{LA CONSTITUCION DE 1967}

La Constitución de la República del Paraguay, sancionada por la Convención Nacional Constituyente el 25 de agosto de 1967, con la participación de todos los partidos políticos democráticos, establece un Estado con una organización eficaz, consagrando derechos, garantías y obligaciones en concordancia con el perfil y la tradición histórica bien definida del pueblo paraguayo.

El artículo $10^{\circ}$ de la Constitución determina que «el Paraguay es y será siempre una Nación libre e independiente». Se constituye en una República unitaria e indivisible. 
La parte dogmática de la Constitución establece los derechos, garantías y obligaciones, enalteciendo la personalidad humana y el derecho del hombre como persona a ser protegido por el Estado en su vida, su integridad física, su libertad, su seguridad, su propiedad, su honor y su reputación (art. 50). Consagra esta Ley fundamental, en capítulos independientes bien definidos, los derechos individuales, los derechos sociales, los derechos económicos, los derechos de los trabajadores y los derechos políticos. En el capítulo de Derechos individuales determina, entre otros principios, lo siguiente: a) que todos los habitantes de la República tienen derecho al libre desenvolvimiento de su personalidad, sin otras limitaciones que las derivadas del derecho de terceros y del orden público y social; b) la igualdad de derechos civiles y políticos de la mujer, cuyos deberes correlativos serán establecidos en la Ley atendiendo a los fines del matrimonio y la unidad en la familia; c) el principio de la inviolabilidad de la defensa, así como que ninguna persona puede ser condenada sin juicio previo, que se funda en Ley anterior al hecho del proceso y juzgado por Tribunales especiales.

En los Derechos sociales el Estado reconoce en la familia la célula fundamental de la sociedad y promueve el constante mejoramiento de su situación moral, cultural, económica y social. Se protege, además, la educación y cultura y la salud. En lo atinente a los Derechos económicos se consagra que el Estado debe promover el desarrollo económico mediante la organización racional de los recursos disponibles con el objeto de impulsar el crecimiento dinámico de la economía nacional, crear nuevas fuentes de trabajo y de riqueza y asegurar el bienestar general. El desarrollo se fomentará sobre la base de programas globales fundados en principios de justicia social que aseguren a todos una existencia compatible con la dignidad humana. La iniciativa privada es estimulada, y se garantiza la propiedad privada, cuyo contenido y límites serán fijados por la Ley, atendiendo a su función económica y social. Así, también se establece que el capital como factor de desarrollo debe cumplir una finalidad económica y social en armonía y recíproca cooperación con el trabajo. El Estado promoverá la formación y consolidación del capital nacional y favorecerá la inversión del capital extranjero en actividades productivas, como complemento necesario para el desarrollo nacional. Asimismo, siguiendo los postulados americanistas de los próceres de la independencia paraguaya, se consigna que el Estado favorecerá el proceso de integración de los países la- 
tinoamericanos, para acelerar su desarrollo equilibrado y aumentar el bienestar común, en función de los intereses de la República y sin detrimento de su soberanía.

En el capítulo atinente al Derecho de los trabajadores «queda proscripta la explotación del hombre por el hombre», siendo el trabajo objeto de protección especial y no estando sujeto a otras condiciones que las establecidas para mejorar la situación material, moral e intelectual del trabajador.

\section{DIVISION DE PODERES}

Las funciones de legislación, administración y jurisdicción se hallan divididas, pero interdependientes las unas de las otras, con el objeto de una realización armoniosa de las funciones estatales.

\section{PODER LEGISLATIVO}

El Poder legislativo de la Nación es ejercido por un Congreso compuesto de dos Cámaras, una de Senadores y otra de Diputados. Las Leyes pueden tener origen en una $u$ otra Cámara del Congreso, mediante proyectos presentados por cualquiera de sus miembros, salvo aquellos cuya iniciativa corresponde al Poder ejecutivo o a una Cámara determinada, por mandato expreso de la Constitución.

Todo proyecto de Ley debe ser presentado con exposición de motivos.

\section{PODER EJECUTIVO}

El capítulo VIII, numeral 1, organiza el Poder ejecutivo y sus atribuciones, los Ministros de dicho Poder y el Consejo de Estado. El Poder ejecutivo es desempeñado por un ciudadano con el título de Presidente de la República del Paraguay. El despacho de los negocios de la República está a cargo de los Ministros del Poder ejecutivo. 


\section{PODER JUDICIAL}

El Poder judicial de la República es ejercido por una Corte Suprema de Justicia, compuesta de cinco miembros, y por los Tribunales y Juzgados que establezca la Ley. La Corte Suprema de Justicia ejerce la Superintendencia de todos los órganos del Poder judicial, con potestad disciplinaria. Entiende en instancia única en las contiendas de jurisdicción y de competencia, conforme a la Ley.

Queda garantizada la independencia del Poder judicial, y «sólo él puede conocer y decidir en los actos de carácter contencioso».

\section{Tribunal de Cuentas}

Queremos destacar muy especialmente que el Tribunal de Cuentas, denominado también de lo Contencioso-administrativo, es un Tribunal de jerarquía constitucional, estando dividido en dos Salas. La primera tiene competencia exclusiva en el control contenciosoadministrativo; la segunda, en el control de las cuentas de inversiones del Presupuesto general de la Nación, sobre cuya ejecución informará anualmente al Poder ejecutivo y a la Cámara de Diputados. La Ley podrá ampliar sus atribuciones.

\section{RECURSO DE INCONSTITUCIONALIDAD}

El artículo 200 establece la facultad de la Corte Suprema de Justicia de declarar la inconstitucionalidad de las Leyes y la inaplicabilidad de las disposiciones contrarias a la Constitución en cada caso concreto y en fallo que sólo tendrá efecto con relación a ese caso. El procedimiento podrá iniciarse por acción ante la Corte Suprema de Justicia, y por excepción en cualquier instancia, y se elevarán sus antecedentes a dicha Corte. $\mathrm{El}$ incidente no suspende el juicio, que prosigue hasta el estado de sentencia.

\section{JERARQUTA DE LOS GRGANOS JUDICIALES}

La nueva Ley número 879, de 2 de diciembre de 1981, que establece el Código de Organización Judicial, determina la jerarquía de los órganos del Poder judicial en la siguiente forma: 
- La Corte Suprema de Justicia.

- El Tribunal de Cuentas.

- Los Tribunales de Apelación.

- Los Juzgados de Primera instancia.

- La Justicia de Paz letrada.

- Los Juzgados de Instrucción en lo penal; y

- Los Jueces-árbitros y Arbitradores.

\section{El Ministerio PÚblico}

El Ministerio público es ejercido por el Fiscal general del Estado, por Agentes fiscales y por Procuradores fiscales, cuyas atribuciones respectivas están reglamentadas en el Código de Organización Judicial.

El Ministerio público es un órgano complementario y auxiliar de la Justicia. Es una magistratura independiente designada por el Poder ejecutivo.

Sintetizando, podemos decir que la Constitución promulgada el 25 de agosto de 1967, siguiendo los principios de MoNTESQuieu y de la Constitución de los Estados Unidos de América, consta de un preámbulo, una parte dogmática y otra orgánica. Son en total 239 artículos, agrupados en once capítulos y uno adicional.

\section{EL MUNICIPIO PARAGUAYO}

El distinguido profesor Daniel-Hugo Martins (9) define al Municipio "como institución político-administrativa-territorial, basada en la vecindad, organizada jurídicamente dentro del Estado, para satisfacer las necesidades de vida de la comunidad local, en coordinación con otros Entes territoriales y servicios estatales».

Nuestra intención es sólo presentar un esquema de las normas del Derecho municipal paraguayo, integrantes del Derecho administrativo, en cuanto se refiere a la organización municipal. El profesor Salvador Villagra MafFiodo (10) especifica lo que debe entenderse

(9) Daniel-Hugo MaRTins: El Municipio contemporáneo, Uruguay, pág. 56.

(10) Salvador Villagra MAfFIodo: Principios de Derecho Administrativo, Asunción, 1981, pág. 354. 
por Administración general y municipal. La Administración general, en un sentido amplio, comprende a la Administración en la que están incluidos los tres Poderes del Estado; mientras que con un sentido restringido dicha «Administración pública» es sólo comprensiva de las actividades propias del Poder ejecutivo, excluyendo las del Poder legislativo y judicial.

Villagra MafFiodo sostiene que «no cabe duda de que la Administración local o municipal está incluida en el concepto amplio de 'Administración pública', puesto que la Institución municipal está integrada y subordinada al Estado».

Esta delimitación es sumamente importante, ya que el artículo 180, inciso 1, atribuye al Presidente de la República la Administración general del país.

Por ello, es necesario en primer lugar ubicar a la Administración municipal dentro del Ordenamiento jurídico constitucional de los Poderes del Estado. Por la prescripción constitucional citada más arriba, ninguna repartición pública organizada bajo el ámbito del Poder ejecutivo, ya sea ella con descentralización de competencia o con descentralización de personalidad, pueden escapar del control ejercido por el Presidente de la República. Ahora bien: ies o no la «Administración municipal» integrante de la «Administración general» en el concepto estricto, de acuerdo al Derecho paraguayo? El profesor VillaGRA MAFFIodo contesta acertadamente este interrogante al decir: «a nuestro juicio, la Administración municipal no está incluida en la 'Administración general del País', que tiene a su cargo el Poder ejecutivo. La Constitución reconoce expresamente la autonomía municipal y prescribe taxativamente los casos de intervención del Poder ejecutivo, de modo que la Ley no puede disponer libremente acerca de estas intervenciones en los Municipios, como puede hacerlo respecto de las demás reparticiones de la 'Administración general'. La Administración municipal está separada a este efecto de la Administración general del País, y si una Ley quiere comprenderla con algún otro fin, como por ejemplo el de planes de fomento o de restricciones administrativas de carácter general, debe disponerlo así expresamente para evitar toda duda». 


\section{LA AUTONOMIA MUNICIPAL EN LA CONSTITUCION}

La Constitución paraguaya consagra la autonomía municipal cuando en el artículo 17 dice textualmente: «Queda reconocida la autonomía municipal. La Ley determinará las modalidades con que dicha autonomía será garantizada a los Municipios, tanto en el orden político como en el jurídico, el económico y el administrativo. Comprenderá esencialmente la elección y designación de sus autoridades; la libre gestión en las materias de su competencia; la determinación de sus bienes y los requisitos y limitaciones para disponer de ellos, así como la de sus ingresos y la forma de recaudarlos e invertirlos; la responsabilidad del gobierno municipal, y los recursos contra sus resoluciones".

Es necesario señalar que la Constitución de 1967 es la primera en el Paraguay que consagra la autonomía municipal. Antes de la sanción de esta Constitución sólo la Ley otorgó autonomía a las Municipalidades, no obstante que el Cabildo colonial tuvo gran tradición en la Provincia del Paraguay. Las Constituciones de 1870 y 1940 delegaban al Poder legislativo la atribución de legislar la organización municipal. De allí que estaba reservada a la Ley el establecimiento de la autonomía municipal, que así como podía crear podía también suprimir.

\section{LEYES MUNICIPALES}

La primera Ley Orgánica de las municipales que organiza en la ciudad de Asunción y en las villas y pueblos fue dictada el 7 de julio de 1882. Si bien la Municipalidad de la capital fue restablecida por Ley del 15 de julio de 1871, en febrero del año siguiente se suspendió su aplicación, pues las condiciones en que había quedado la ciudad capital después de la guerra eran inapropiadas para poner en marcha la Oficina municipal.

\section{LA LEY NƯMERO 915}

La Ley número 915, del 1 de septiembre de 1927, organiza las Municipalidades de todo el país y tiene sus fuentes directas en las Leyes Orgánicas de la República Argentina y de los Estados Unidos de América. 


\section{LEY NÚMERO 222 ORGÁNICA MUNICIPAL}

La Ley vigente fue promulgada el 30 de julio de 1954. Dicha Ley expresa que el Municipio es un conjunto de vecinos que ocupan un determinado territorio y que reúne los requisitos señalados en esta Ley.

\section{PRESCRIPCIONES CONSTITUCIONALES}

El artículo 18 de la Constitución determina que será privativo de los Municipios la competencia para el gobierno y la administración de los intereses comunales en las siguientes materias: urbanismo, abasto, educación y cultura, asistencia sanitaria y social, montepío, tránsito, turismo, inspección y policía municipal.

Expresa la Constitución que la Ley podrá autorizar la creación del servicio de carácter nacional o departamental en la jurisdicción de los Municipios. Esta creación en la mayor parte tendría un carácter subsidiario, en razón de que los principales servicios públicos son prestados por empresas públicas estatales, con descentralización de personalidad jurídica.

La Constitución también determina los casos de intervención del Poder ejecutivo, que son los siguientes:

1) A solicitud de la Junta municipal.

2) Por desintegración de la Junta municipal que imposibilite su funcionamiento.

3) Cuando se produzca déficit presupuestario durante dos ejercicios anuales consecutivos; o

4) En caso de grave irregularidad determinada taxativamente por la Ley.

La intervención no se prolongará por más de noventa días.

En casos de desintegración, los comicios para constituir las nuevas autoridades electivas se realizarán dentro de dicho plazo. Si de la intervención resultare el cese de dichas autoridades, las elecciones para sustituirlas se llevarán a cabo dentro de sesenta días contados desde la referida cesantía. 


\section{ELEMENTOS ESENCIALES}

Concordamos plenamente con MARTINS cuando señala los elementos esenciales necesarios para que exista un Municipio, que a su criterio son los siguientes:

«a) La existencia de un grupo social humano: el vecindario o población afincada en un lugar.

b) Un territorio, es decir, una circunscripción territorial delimitada (llámese Pago, Parroquia, Distrito, Partido, Departamento, Pueblo o Ciudad).

c) Una organización político-administrativa, con personalidad jurídica dentro del Estado.

d) Un cúmulo de atribuciones sobre determinadas materias (competencia).

e) Un Ordenamiento jurídico (conjunto de principios y reglas de Derecho, creadas por el Estado o por el mismo Municipio) que regula su estructura, su funcionamiento, su actividad y las relaciones jurídicas con sus habitantes, con el Estado o con otras personas jurídicas públicas o privadas.

f) Un patrimonio propio (conjunto de bienes pertenecientes a la comunidad local)».

Concordamos con Dromi (11) cuando dice que el Municipio está entrañablemente ligado - unido- a un fenómeno moderno, generado por el crecimiento industrial: el urbanismo. Con acierto, DromI reseña lo que provocó el paso de la civilización agraria a la civilización industrial: el nacimiento de los grandes centros poblados, metrópolis, megápolis, etc., que se han constituido, dice, «en un verdadero desafío al hombre de estos tiempos». Dromi lanza un llamado cuando dice que «debemos tratar de salvar al individuo, antes de que sea demasiado tarde, del acecho de una industrializaciónmecanización que lo prive de iniciativa e imaginación y aglutine en condiciones de vida no siempre recomendables para los que pensamos que el bien común y la justicia social son valores realizables aquí y ahora».

(11) José-Roberto Dromi: Federalismo y Municipio, Buenos Aires, 1980, pág. 74. 


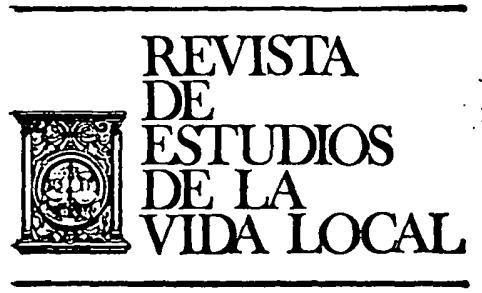

\section{CRONICAS}


REVL-1983, núm. 219. CHASE PLATE, LUIS-ENRIQUE. ESTADO Y MUNICIPIO.

REVL-1983, núm. 219. CHASE PLATE, LUIS-ENRIQUE. ESTADO Y MUNICIPIO. 